\title{
At the crossroads of oxygen and iron sensing: hepcidin control of HIF-2 $\alpha$
}

\author{
Frank S. Lee
}

Department of Pathology and Laboratory Medicine, Perelman School of Medicine, University of Pennsylvania, Philadelphia, Pennsylvania, USA.

\begin{abstract}
Hepcidin is the master regulator of iron metabolism. It plays a key role in the regulation of iron transport across the duodenal epithelium, which in turn is dependent on the oxygen-regulated transcription factor hypoxia-inducible factor $2 \alpha$ (HIF-2 $\alpha$ ). In this issue of the JCI, Schwartz and colleagues show that duodenal HIF- $2 \alpha$ is itself regulated by hepcidin, thereby indicating that this transcription factor is not only regulated by oxygen, but also by iron. This work indicates that the crosstalk between liver hepcidin and intestinal HIF-2 $\alpha$ plays an important role during iron overload, systemic iron deficiency, and anemia.
\end{abstract}

\section{Hepcidin}

Hepcidin is produced primarily in the liver by hepatocytes, which sense plasma iron through a complex network of membrane-bound proteins that include the HLA class I-like protein HFE, transferrin receptor 1 (TFR1), transferrin receptor 2 (TFR2), hemojuvelin (HJV), and proteins of the BMP receptor family $(1,2)$. The BMP receptors regulate the activity of the SMAD family of transcription factors, which are key regulators of hepcidin gene transcription. High levels of plasma iron result in increased hepcidin transcription and increased circulating levels of hepcidin. Conversely, low levels of plasma iron result in decreased hepcidin transcription and decreased circulating levels of hepcidin.

The key function of hepcidin is to bind to ferroportin (FPN), the only known mammalian cellular exporter of iron (3), and induce its degradation in cells that include duodenal enterocytes, macrophages, and hepatocytes. Hence, iron-replete conditions (resulting in high levels of hepcidin) suppress FPN protein expression, leading to cellular retention of iron (Figure 1A). Conversely, low iron conditions (low levels of hepcidin) lead to FPN stabilization, resulting in efflux of iron into the circula- tion. This allows the systemic mobilization of iron. The largest consumers of iron are $\mathrm{RBC}$, in which iron is incorporated as heme into the oxygen carrier hemoglobin.

\section{Hypoxia-inducible factor}

Red cell mass is critically controlled by hypoxia-inducible factor (HIF), which is a key regulator of oxygen homeostasis. HIF is a heterodimeric transcription factor consisting of an $\alpha$ subunit, HIF- $\alpha$, and a $\beta$ subunit, HIF- $\beta$ (4-6). The two most extensively studied HIF- $\alpha$ isoforms are HIF-1 $\alpha$ and HIF- $2 \alpha$ (also known as EPAS1). HIF is primarily controlled by oxygen-regulated degradation of the $\alpha$ subunit. Under normoxic conditions, a family of three oxygen-dependent, iron-containing prolyl hydroxylases, PHD1, PHD2, and PHD3 (also known as EGLN2, EGLN1, and EGLN3, respectively), constitutively and site specifically hydroxylate key prolyl residues on HIF- $1 \alpha$ and HIF- $2 \alpha$. This provides a recognition motif for the von HippelLindau tumor suppressor protein, a component of an E3 ubiquitin ligase complex that targets HIF- $\alpha$ for degradation. Under hypoxic conditions, this modification is arrested, leading to the stabilization of HIF- $\alpha$ and activation of HIF target genes.

Related Article: p. 336

Conflict of interest: The author has declared that no conflict of interest exists.

Reference information: / Clin Invest. 2019;129(1):72-74. https://doi.org/10.1172/JCI125509.

The HIF pathway operates in essentially all tissues of the body. Its use, however, displays cell-and organ-dependent differences. For example, in specialized interstitial cells of the renal cortex and in hepatocytes, a critical HIF- $2 \alpha$ gene target is the hormone erythropoietin (EPO) (7, 8). EPO stimulates the expansion of red cell mass in an oxygen-regulated manner, thereby improving oxygen delivery to tissues of the body. Moreover, as with HIF- $1 \alpha$ and $-2 \alpha$, the three PHDs are not equivalent proteins. For example, PHD2 possesses an $\mathrm{N}$-terminal zinc finger, absent from PHD1 and PHD3, that promotes recruitment to the HSP90 pathway to facilitate hydroxylation of HIF- $\alpha$, an HSP90 client (9-11).

\section{HIF and iron}

Red cell mass and iron delivery must be tightly coordinated. Indeed, after hypoxia initiates EPO-induced erythropoiesis, erythroid progenitors secrete erythroferrone, a hormone that suppresses hepcidin production, thereby stimulating iron mobilization (12). Decreased hepcidin not only leads to FPN stabilization, but also increased transcription of genes encoding for duodenal cytochrome B (DCYTB), divalent metal transporter 1 (DMT1), and FPN itself in duodenal enterocytes (Figure $1 B)$. HIF- $2 \alpha$ plays a critical role in mediating this process (13-15). DCYTB reduces $\mathrm{Fe}$ (III) to duodenal lumen Fe(II), thereby allowing its transport across the apical surface by DMT1. The FPN, DCYTB, and $D M T 1$ genes are all targets of HIF- $2 \alpha$ (15).

In the present JCI study, Schwartz and colleagues (16) pursued the finding that duodenal cells employ HIF- $2 \alpha$, ordinarily an oxygen-sensitive transcription factor, to regulate genes central to iron metabolism. To separate hypoxia from hepcidininduced effects, they first examined mice with an inducible liver conditional knockout of hepcidin and observed the activation of HIF- $2 \alpha$ and HIF- $2 \alpha$ target genes (but, interestingly, not HIF- $1 \alpha$ or HIF- $1 \alpha$ target genes) in duodenal enterocytes. For 
A Iron-replete

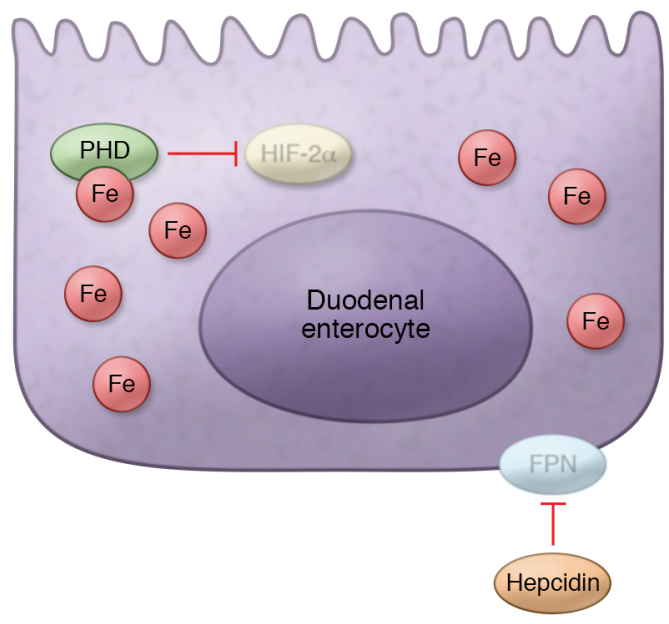

B Iron-deficient

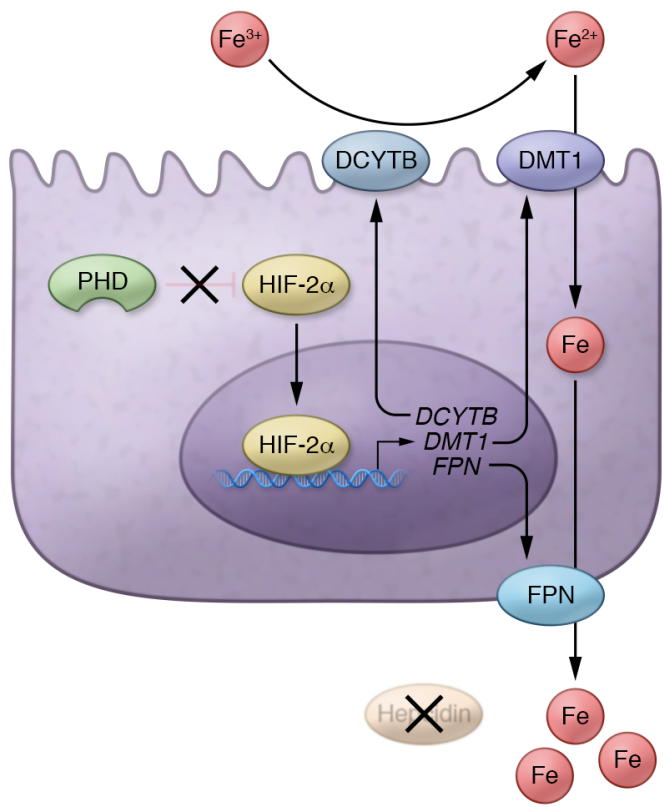

Figure 1. HIF-2 $\alpha$ regulation by hepcidin in duodenal enterocytes. (A) Under iron-replete conditions, hepcidin constitutively induces FPN degradation, leading to enterocyte retention of iron ( $\mathrm{Fe}$ ), high PHD activity, and constitutive degradation of HIF-2 $\alpha$. (B) Under iron-deficient conditions, lack of hepcidin leads to stabilization of FPN, efflux of iron, and lowered intracellular iron concentration. This leads to decreased activity of iron-dependent PHD, stabilization of HIF-2 $\alpha$, and activation of the HIF-2 $\alpha$ target genes DCYTB, DMT1, and FPN. DCYTB reduces $\mathrm{Fe}^{3+}$ to $\mathrm{Fe}^{2+}$, which in turn is transported from the apical surface into the enterocyte by DMT1. FPN promotes the transport of iron out the enterocyte through its basolateral surface.

FPN, DCYTB, DMT1, and TFRC, upregulation was observed at both mRNA and protein levels.

Since hepcidin acts through FPN, the authors then examined mice with an inducible intestinal conditional knockout of Fpn and found that it blocked both low iron- and anemia-induced activation of HIF- $2 \alpha$, demonstrating an essential role for FPN in this process. This raises the possibility that intracellular iron levels may modulate HIF-2 levels. Importantly, inducible intestinal conditional knockout of Dmt1, a means to lower iron levels in the enterocyte, results in duodenal activation of HIF- $2 \alpha$. In cell culture studies, both a human HEK293 cell line (17) and a rat IEC-6 intestinal cell line with inducible expression of FPN display HIF-2 $\alpha$ stabilization upon FPN induction. Moreover, a PHD reporter construct consisting of a hydroxylation substrate domain fused to luciferase shows stabilization in both cell lines upon FPN induction (16).

\section{Regulation of HIF-2 $\alpha$ by iron}

Taken together, the results of the study by Schwartz and colleagues (16) lead to the intriguing conclusion that in the hepcidin pathway, the oxygen-regulated HIF- $2 \alpha$ transcription factor is, in fact, regulated by iron (Figure 1). Specifically, because iron is an obligatory cofactor for PHD, low iron leads to low PHD activity. Thus, the PHDs can be thought of as key integrators of signals that extend beyond oxygen and include iron. Of note, the PHDs are also responsive to agents that include ROS and Krebs cycle intermediates. In addition, the data indicate that the duodenal epithelial cell has a distinctive configuration of the HIF pathway that allows it to sense iron (in addition to oxygen) and thereby regulate genes of the iron pathway (FPN, DCYTB, DMT1). This is reminiscent of the manner in which specialized renal cells specifically employ the PHD2/HIF-2 $\alpha$ axis to control EPO production in response to changing oxygen concentrations (7, 18-21).

The study by Schwartz et al. (16) provides new insights into the role of the HIF pathway in iron metabolism. This leads to the mechanistic question of whether iron is simply passively lost from the PHDs upon iron deprivation. In this regard, it might be noted that PHD2, the central PHD isoform, has a high affinity for iron, at least in vitro (22). Alternatively, low intracellular iron levels could potentially impair the assembly of PHD from iron and the PHD apoenzyme. More broadly, the PHDs are members of a larger family of 2-oxoglutarate-dependent dioxygenases (23), raising the question of whether other such enzymes may be regulated by iron availability in a similar manner.

One enzyme that is well known to be regulated by intracellular iron levels is cytosolic aconitase (c-aconitase) (24). In its native form, the iron-containing c-aconitase catalyzes the interconversion of citrate and isocitrate. Upon iron depletion, it converts to iron-regulatory protein 1 (IRP1), an RNA-binding protein that binds to iron response elements (IREs). Remarkably, one such IRE that inhibits translation is located in the $5^{\prime}$-UTR of the HIF2A mRNA itself, and it provides a means by which HIF- $2 \alpha$ activity can be attenuated under conditions in which iron stores are depleted (25). The authors suggest that IRP may limit the extent of HIF-2 $\alpha$ activity upon iron deficiency.

\section{Preference for HIF-2 $\alpha$ over HIF-1 $\alpha$}

A relevant issue is the mechanism by which HIF- $2 \alpha$ is selectively activated over HIF- $1 \alpha$ in these cells, since both are posttranslationally regulated by the same family of 
iron-containing PHDs. Another important question is how broadly this iron-sensing mechanism is employed. The present studies have focused on the regulation of HIF$2 \alpha$ by hepcidin in duodenal enterocytes. In addition to these cells, macrophages and hepatocytes provide stores of iron, and like duodenal enterocytes, these cells can express FPN (1). In their animal models, Schwartz and colleagues did not observe changes in HIF-2 $\alpha$ target gene expression in the spleen, an organ harboring FPNexpressing macrophages, which may suggest differences in how HIF- $2 \alpha$ responds to iron deficiency in different cell types. It will be of interest to determine the mechanistic basis of any such differences.

\section{Acknowledgments}

Work in the author's laboratory is supported by NIH grants R01-DK104796 and R33HL120751, and by National Science Foundation (NSF) grant BCS-1638642.

Address correspondence to: Frank S. Lee, Department of Pathology and Laboratory Medicine, Perelman School of Medicine, University of Pennsylvania, 605 Stellar Chance Labs, 422 Curie Blvd., Philadelphia, Pennsylvania 19104, USA. Phone: 215.898.4701; Email: franklee@ pennmedicine.upenn.edu.

\footnotetext{
1. Coffey R, Ganz T. Iron homeostasis: an anthropocentric perspective. J Biol Chem. 2017;292(31):12727-12734
}

2. Muckenthaler MU, Rivella S, Hentze MW, Galy B. A red carpet for iron metabolism. Cell. 2017;168(3):344-361.

3. Nemeth E, et al. Hepcidin regulates cellular iron efflux by binding to ferroportin and inducing its internalization. Science. 2004;306(5704):2090-2093.

4. Semenza GL. Oxygen sensing, hypoxia-inducible factors, and disease pathophysiology. Annu Rev Pathol. 2014;9:47-71.

5. Kaelin WG Jr., Ratcliffe PJ. Oxygen sensing by metazoans: the central role of the HIF hydroxylase pathway. Mol Cell. 2008;30(4):393-402.

6. Majmundar AJ, Wong WJ, Simon MC. Hypoxiainducible factors and the response to hypoxic stress. Mol Cell. 2010;40(2):294-309.

7. Lee FS, Percy MJ. The HIF pathway and erythrocytosis. Annu Rev Pathol. 2011;6:165-192.

8. Haase VH. Regulation of erythropoiesis by hypoxia-inducible factors. Blood Rev. 2013;27(1):41-53.

9. Song D, Li LS, Heaton-Johnson KJ, Arsenault PR, Master SR, Lee FS. Prolyl hydroxylase domain protein 2 (PHD2) binds a Pro-Xaa-Leu-Glu motif, linking it to the heat shock protein 90 pathway. J Biol Chem. 2013;288(14):9662-9674.

10. Arsenault PR, Song D, Chung YJ, Khurana TS, Lee FS. The zinc finger of prolyl hydroxylase domain protein 2 is essential for efficient hydroxylation of hypoxia-inducible factor $\alpha$. Mol Cell Biol. 2016;36(18):2328-2343.

11. Sinnema M, et al. Loss-of-function zinc finger mutation in the EGLN1 gene associated with erythrocytosis. Blood. 2018;132(13):1455-1458.

12. Kautz L, Jung G, Valore EV, Rivella S, Nemeth $\mathrm{E}, \mathrm{Ganz}$ T. Identification of erythroferrone as an erythroid regulator of iron metabolism. Nat Genet. 2014;46(7):678-684.

13. Shah YM, Matsubara T, Ito S, Yim SH, Gonzalez FJ. Intestinal hypoxia-inducible transcription factors are essential for iron absorption following iron deficiency. Cell Metab. 2009;9(2):152-164.

14. Mastrogiannaki M, Matak P, Keith B, Simon
MC, Vaulont S, Peyssonnaux C. HIF-2 $\alpha$, but not HIF-1 $\alpha$, promotes iron absorption in mice. J Clin Invest. 2009;119(5):1159-1166.

15. Ramakrishnan SK, Shah YM. Role of intestinal HIF- $2 \alpha$ in health and disease. Annu Rev Physiol. 2016;78:301-325.

16. Schwartz AJ, et al. Hepatic hepcidin/intestinal HIF- $2 \alpha$ axis maintains iron absorption during iron deficiency and overload. J Clin Invest. 2019;129(1):336-348.

17. Qiao B, et al. Hepcidin-induced endocytosis of ferroportin is dependent on ferroportin ubiquitination. Cell Metab. 2012;15(6):918-924.

18. Percy MJ, et al. A family with erythrocytosis establishes a role for prolyl hydroxylase domain protein 2 in oxygen homeostasis. Proc Natl Acad Sci U S A. 2006;103(3):654-659.

19. Percy MJ, et al. A gain-of-function mutation in the HIF2A gene in familial erythrocytosis. $N \mathrm{Engl}$ JMed. 2008;358(2):162-168.

20. Gruber M, Hu CJ, Johnson RS, Brown EJ, Keith B, Simon MC. Acute postnatal ablation of Hif- $2 \alpha$ results in anemia. Proc Natl Acad Sci US A. 2007;104(7):2301-2306.

21. Kapitsinou PP, et al. Hepatic HIF-2 regulates erythropoietic responses to hypoxia in renal anemia. Blood. 2010;116(16):3039-3048.

22. McNeill LA, et al. Hypoxia-inducible factor prolyl hydroxylase 2 has a high affinity for ferrous iron and 2-oxoglutarate. Mol Biosyst. 2005;1(4):321-324.

23. Losman JA, Kaelin WG. What a difference a hydroxyl makes: mutant IDH, (R)-2hydroxyglutarate, and cancer. Genes Dev. 2013;27(8):836-852.

24. Muckenthaler MU, Galy B, Hentze MW. Systemic iron homeostasis and the iron-responsive element/iron-regulatory protein (IRE/IRP) regulatory network. Annu Rev Nutr. 2008;28:197-213.

25. Sanchez M, Galy B, Muckenthaler MU, Hentze MW. Iron-regulatory proteins limit hypoxiainducible factor- $2 \alpha$ expression in iron deficiency. Nat Struct Mol Biol. 2007;14(5):420-426. 